\title{
REVIEW
}

\section{Chronic obstructive pulmonary disease - diagnosis and classification of severity}

\author{
P J Viviers, ${ }^{1} \mathrm{MB}$ ChB, MMed (Int), Cert Pulmonology (SA); \\ R N van Zyl-Smit, ${ }^{2}$ MB ChB, MRCP (UK), FCP (SA), Dip HIV Man (SA), MMed, Cert Pulmonology (SA), PhD \\ ${ }^{1}$ Private Practice, Life-Wilgers Hospital, Pretoria, South Africa \\ ${ }^{2}$ UCT Lung Institute, Division of Pulmonology, Department of Medicine, Faculty of Health Sciences, University of Cape Town, South Africa
}

Corresponding author: P J Viviers (drpjv@live.com)

\begin{abstract}
Chronic obstructive pulmonary disease (COPD) is a common, progressive and preventable non-communicable respiratory disorder. It is often confused with asthma and poorly understood by many lay people. The primary cause of COPD is tobacco smoking, but in the South African (SA) context, biomass fuel exposure/household pollution, tuberculosis, HIV and mining exposure are additional important causes. There is a very high prevalence of COPD in SA and it is the third leading cause of mortality globally.

The diagnosis of COPD is based predominantly on symptoms, i.e. progressive shortness of breath and cough in a patient with risk factors - usually smoking. Lung function testing is required to formally make the diagnosis, which places a significant hurdle in correctly identifying COPD in SA, given the limited access to spirometry in many areas. Spirometry is also required to grade the severity of lung function obstruction.

Severity assessment, which is used to plan a management strategy (predominantly bronchodilators with inhaled steroids in severe cases), combines symptoms, lung function and exacerbations. Based on these 3 factors, a patient can be categorised into 1 of 4 groups and appropriate management instituted. Additional comorbidities, particularly cardiovascular and mental illness, should also be evaluated.

Early identification of COPD, with further avoidance of an aetiological cause such as smoking, is key in preventing disease progression. Appropriate therapy, comprising non-pharmacological and pharmacological interventions and based on a comprehensive severity assessment, should result in symptom improvement and reduced risk for exacerbations.
\end{abstract}

S Afr Med J 2015;105(9):786-788. DOI:10.7196/SAMJnew.8421

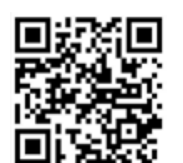

Chronic obstructive pulmonary disease (COPD) is a common, progressive and preventable disorder of the airways. It is often poorly understood by patients, and frequently referred to as 'asthma' or emphysema, which adds to the confusion. It is characterised by predominantly fixed airway obstruction as a result of exposure to inhaled noxious environmental particles or gases, e.g. cigarette smoke, biomass fuels, occupational exposure. ${ }^{[1]}$ The predominant symptoms are progressive dyspnoea, cough and wheeze, interlaced with periods of acute worsening or so-called exacerbations.

COPD is a prevalent condition (329 million people are affected worldwide), with an increasing incidence. Currently, the World Health Organization (WHO) ranks COPD as the fourth most important cause of death due to non-communicable disease worldwide, which is predicted to rise to the third place by $2020 .^{[2]}$ In South Africa (SA) there are limited data, but data from Cape Town estimated the prevalence to be high (>20\%); compared with other countries in the BOLD study, Cape Town ranked highest. ${ }^{[3]}$ The population studied was not representative of the entire country, and there is no clear estimate of how prevalent COPD is. There are also no substantial data from the rest of Africa.

Compared with COPD, asthma, a chronic inflammatory disease of the airways, features highly variable airflow obstruction (i.e. clinically suggested by a positive bronchodilator response) in most cases, leading to episodes of cough, wheeze and dyspnoea, but usually with a return to normal baseline lung function between these acute 'attacks'. The focus of this review is the correct diagnosis of COPD. It is important to differentiate the condition from asthma as the progression of disease, expectation of patient and doctor, and management are different.
It is not always easy to distinguish COPD from asthma because of shared clinical features. The term asthma-COPD overlap syndrome (ACOS) has been coined to identify this group, which includes features of both conditions. ${ }^{[4]}$ It is the subject of much debate and beyond the scope of this review, but if in doubt it is considered safer to treat the patient as if they are asthmatic. This review focuses on the diagnosis and severity evaluation of COPD and relies heavily on the South African Thoracic Society Guidelines ${ }^{[5]}$ and the updated GOLD strategy document and teaching slides. ${ }^{[1]}$

\section{Diagnosis of COPD}

A complete history and thorough clinical examination are vital and cannot be substituted by any other measure when considering a diagnosis of COPD. A progressive decline in respiratory function, characterised by dyspnoea or worsening shortness of breath on effort (e.g. walking on the level or climbing stairs), cough and wheeze, interlaced by periods of intermittent worsening (or exacerbations), in a patient with known risk factors is typical for a diagnosis of COPD. The disease usually presents after the age of 40 years, with a slow progressive onset of symptoms. Importantly, such patients generally do not enjoy a full return to normal functioning over a period of time, despite treatment (as is the case with asthma). A rapid onset of symptoms should raise suspicion of an alternative condition. The second key element is a significant exposure history, specifically to tobacco smoking - generally $>10$ pack-years ( 20 per day for 10 years) for the diagnosis to be considered. If the person started smoking in their teenage years, used dagga and/or methaqualone (Mandrax), COPD may well occur before the age of 40 . One should also enquire about additional exposures such as biomass/indoor air pollution, mining exposure, previous tuberculosis (TB), HIV, and childhood diseases. 
Clinical examination is characterised by hyperinflation, reduced breath sounds and possibly expiratory wheezing. Be certain to look for clubbing and lymphadenopathy, as the risk of cancer is high in heavy smokers. Findings on clinical examination often parallel disease severity in advanced cases; a myriad of clinical signs might be quite obvious (e.g. sarcopenia, pursed-lip breathing, a tripod stance, soft-to-absent breath sounds), but in the earlier stages there might be a paucity of clinical findings. The presence of signs of right heart failure/cor pulmonale (raised jugular venous pressure, hepatomegaly and peripheral oedema) and cardiac arrhythmias (e.g. atrial fibrillation) should be sought, as these require additional management and in most cases prompt referral.

A major stumbling block to the correct diagnosis of COPD is the requirement for spirometry. The use of this technique is recognised by international and local guidelines; without it a diagnosis cannot be confirmed. Airway obstruction is spirometrically defined as the forced expiratory volume in 1 second $\left(\mathrm{FEV}_{1}\right)$ /forced vital capacity (FVC) ratio $<0.7$, with an absolute value of $\mathrm{FEV}_{1}<80 \%$ of predicted for age, gender and height. Clinicians are urged to refer to the published guidelines on the practical use of office spirometry. ${ }^{[6]}$ The post-bronchodilator $\mathrm{FEV}_{1}$ is also used to grade the severity of obstruction, which is important when deciding on management. This is percentage-predicted $\mathrm{FEV}_{1}$, not the severity of the ratio that is used to grade lung function impairment. Lung function may help to distinguish a COPD patient from one with asthma, but reversibility or the lack thereof does not rule out either. Lung function must be viewed against the clinical picture. Reversibility of $>12 \% / 200 \mathrm{~mL}$ occurred in $>50 \%$ of patients enrolled in the large UPLIFT COPD study, but reversibility does not always help to differentiate asthma from COPD. ${ }^{[7]}$

A chest radiograph may depict features of hyperinflation, but does not distinguish between asthma and COPD. The value of an initial chest radiograph during work-up is mainly in screening for additional or alternative lung pathology, such as malignancy, parenchymal disease (pulmonary fibrosis), or previous TB with scarring, but is not a prerequisite to start therapy. High-resolution computed tomography (HRCT) scanning of the chest is excellent for anatomical assessment in COPD, as it can precisely locate and categorise emphysema. Its use is limited by the high cost and lack of general

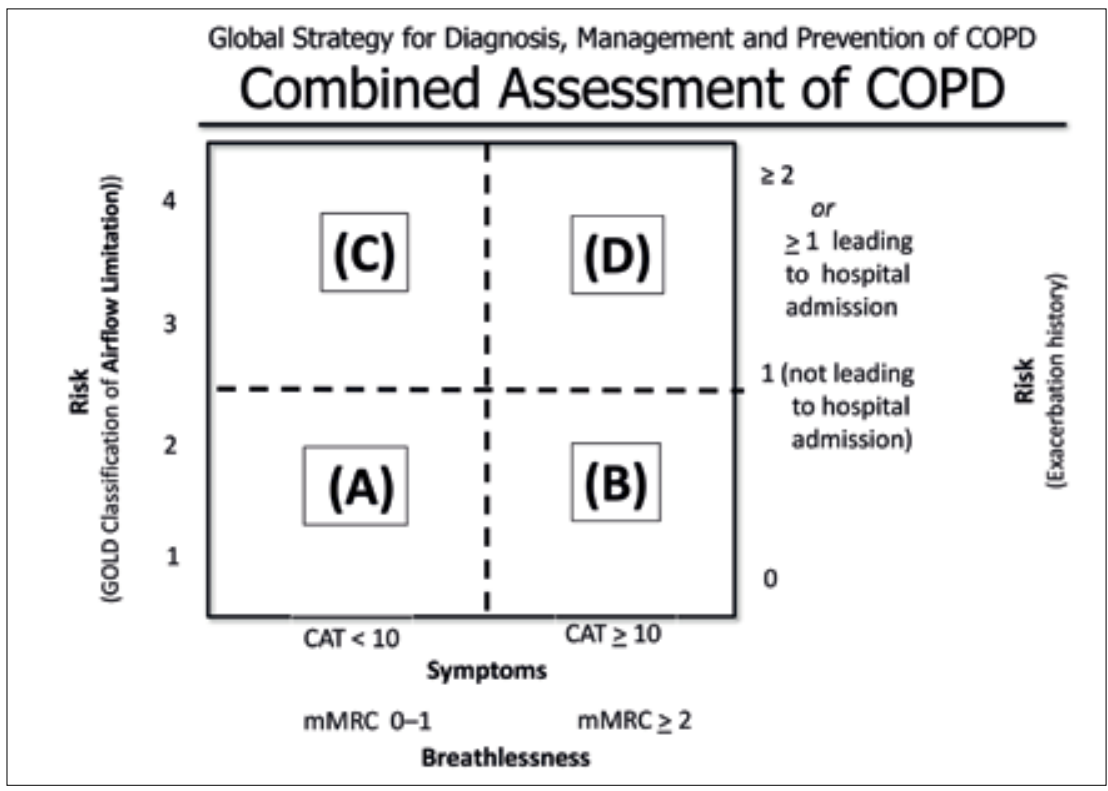

Fig. 1. Global Initiative for Chronic Obstructive Lung Disease classification of COPD severity. Evaluation of symptoms and breathlessness ( $x$-axis), lung function (y-axis, left) and exacerbation history ( $y$-axis, right) provides a combined severity assessment upon which to base a treatment strategy (CAT = COPD assessment test; $m M R C=$ modified Medical Research Council dyspnoea scale; GOLD classification of airflow limitation 1 and $2 \mathrm{FEV}, 250 \%$ predicted, 3 and $4 \mathrm{FEV}$ $<50 \%$ predicted). (From the Global Strategy for Diagnosis, Management and Prevention of COPD 2015, () Global Initiative for Chronic Obstructive Lung Disease (GOLD), all rights reserved. Available from http://www.goldcopd.org.)

availability, and it is not required for the diagnosis of COPD in the vast majority of cases.

Oxygen saturation or arterial blood gas determination is useful in severe disease, where respiratory failure is suspected and long-term home oxygen is being considered. In patients who present very early or have a family history of COPD, consider testing for alpha-1 antitrypsin deficiency.

\section{Assessment of severity}

COPD and asthma are graded according to severity, and management recommendations are based on severity categories. Traditionally, management was decided on by lung function severity alone, but there has been a growing realisation that $\mathrm{FEV}_{1}$ and clinical symptoms correlate poorly; therefore, other factors are integrated into the severity grading. The second conceptual change is that severity is 'graded' rather than 'staged', as patients do not necessarily move linearly from one stage to another over time.

The South African Thoracic Society Guidelines $^{[5]}$ and the GOLD strategy document ${ }^{[1]}$ both incorporate assessments of $\mathrm{FEV}_{1}$ and dyspnoea; however, the presence of exacerbations is prominent in the newer GOLD guidelines. We focus on these as the most current approach to severity assessment. Based on the severity grading, appropriate therapy with bronchodilators and inhaled steroids (in severe cases) may then be instituted. For all grades of severity, smoking cessation, structured exercise plans, good nutrition and vaccination should be considered as part of a holistic treatment plan.

The GOLD 'square box' is the focus of the assessment and may be daunting at first, but once used it is relatively easy (Fig. 1). It is easiest to follow a step-wise approach:

\section{Assess symptoms and breathlessness}

Either the COPD assessment test (CAT) ${ }^{[8]}$ or the modified Medical Research Council (mMRC) dyspnoea scale (Fig. 2) may be used. ${ }^{[1]}$ Patients can fill in the CAT or mMRC form in the waiting room prior to seeing the doctor, saving valuable consultation time. It also allows for objective assessment of symptoms/dyspnoea severity over time. The form is available in several local languages. For the mMRC the cut-off of $\geq 2$ can be simply defined as not being able to keep up with a healthy peer. This assessment places the patient left or right of the centre line (Fig. 1).

\section{Evaluate the severity of lung function} In patients with a post-bronchodilator $\mathrm{FEV}_{1} /$ FVC ratio $<0.70$ :

- GOLD 1: Mild - $\mathrm{FEV}_{1} \geq 80 \%$ predicted

- GOLD 2: Moderate - $\mathrm{FEV}_{1}<80 \%$ to $\geq 50 \%$ predicted 


\section{PLEASE TICK IN THE BOX THAT APPLIES TO YOU (ONE BOX ONLY)}

mMRC Grade 0 . I only get breathless with strenuous exercise.

\author{
mMRC Grade 1. I get short of breath when hurrying on the level \\ or walking up a slight hill.
}

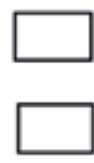

mMRC Grade 2. I walk slower than people of the same age on the level because of breathlessness, or I have to stop for breath when walking on my own pace on the level.

mMRC Grade 3. I stop for breath after walking about 100 meters or after a few minutes on the level.

mMRC Grade 4. I am too breathless to leave the house or I am breathless when dressing or undressing.

Fig. 2. Modified Medical Research Council dyspnoea scale. Patients may review their own shortness of breath grade based on the description provided for each grade. (From the Global Strategy for Diagnosis, Management and Prevention of COPD 2015, (c) Global Initiative for Chronic Obstructive Lung Disease (GOLD), all rights reserved. Available from http://www.goldcopd.org.)

- GOLD 3: Severe - $\mathrm{FEV}_{1}<50 \%$ to $\geq 30 \%$ predicted

- GOLD 4: Very severe - $\mathrm{FEV}_{1}<30 \%$ predicted.

An $\mathrm{FEV}_{1}<50 \%$ predicted places a patient in a category above the horizontal line.

3. Review the occurrence of exacerbations in the past 12 months

An exacerbation is generally considered as a worsening of COPD symptoms, resulting in treatment with corticosteroids and/or antibiotics. More than 2 exacerbations or 1 exacerbation requiring hospitalisation places the patient 'above the line'. Exacerbations are considered more important than lung function in the assessment of risk; thus, their presence places one above the line even if $\mathrm{FEV}_{1}>50 \%$ predicted.

\section{Evaluate for comorbid diseases such as} hypertension, heart failure, diabetes

This is not used to place a patient in a box, but is important for risk evaluation and treatment decisions.

With these elements, the patient can then be 'allocated' to box A, B, C or D. The combined assessment therefore takes the following into consideration: lung function severity, symptoms and exacerbations. The patient should be allocated to the highest risk category, e.g. a patient with an mMRC $>2$, plus 2 exacerbations, but $\mathrm{FEV}_{1}>50 \%$, should be $\mathrm{D}$ - not B. For groups $\mathrm{A}$ and $\mathrm{B}$ long-acting bronchodilators are the primary treatment option, and for groups $\mathrm{C}$ and $\mathrm{D}$ the addition of inhaled steroids in patients with more severe disease. ${ }^{[1]}$

\section{Assessment of comorbidities}

Cardiovascular disease contributes to the majority of deaths in COPD, despite stage or severity, and it is therefore prudent that the medical practitioner evaluate all patients for cardiovascular risk factors. This includes blood pressure measurements, assessment of lipid and glucose metabolism, and electrocardiography, with effort testing where indicated. Treatment of these risk factors contributes to a decreased risk of incident myocardial infarction, stroke and arrhythmias - the major causes of mortality in COPD. ${ }^{[9]}$ Obstructive sleep apnoea is an increasingly common condition, adding to baseline risk, and questionnaires such as the Epworth sleepiness scale ${ }^{[10]}$ may be used to assess risk and determine referral for sleep evaluation.

Psychiatric comorbidity and psychosocial stressorsinfluencepatient behaviour, particularly regarding smoking cessation. The presence of a major depressive disorder decreases continued abstinence rates. ${ }^{[1]}$ Furthermore, psychiatric conditions are prevalent in COPD, as in most chronic disorders, and if left untreated might lead to decreased compliance with management and reduced quality of life. ${ }^{[12]}$

Osteoporosis screening should be done in male and female patients with increased risk, especially if they still smoke. COPD contributes to overall fracture risk, and fractures increase long-term morbidity and mortality. ${ }^{[13]}$ It is therefore essential that this frequently overlooked risk factor is evaluated and managed appropriately.

\section{Conclusion}

A diagnosis of COPD should be suspected in patients who present with slowly progressive shortness of breath, even without a history of smoking. Take a detailed history of exposure to tobacco, dagga, methaqualone and other environmental pollutants. Document the history of exacerbations and identify other comorbid diseases. Clinical examination is helpful to support the diagnosis and rule out other respiratory conditions. Spirometry/lung function tests (pre- and post-FEV ${ }_{1}$ and $\mathrm{FVC}$ ) are required to confirm the diagnosis and aid in severity grading. Radiology is often supportive but not a prerequisite, unless one is screening for lung cancer, and other specialised tests are generally not needed. With this holistic evaluation in place, appropriate decisions on management can be taken. Where symptoms and signs do not match, the disease progression is rapid, or in young patients referral to a specialist may be needed to exclude another unrecognised pulmonary condition.

\section{References}

1. Global Initiative for Chronic Obstructive Lung Disease (GOLD). Global Strategy for Diagnosis, Management and Prevention of COPD. 2015. http://www.goldcopd.org and Prevention of COPD.

2. World Health Organization. The Global Burden of Disease: 2004 World Health Organization.
update. Geneva: WHO, 2008 .

3. Buist AS, McBurnie MA, Vollmer WM, et al. International variation in the prevalence of COPD (the BOLD Study): A population-based prevalence study. Lancet 2007;370(9589):741750 .

4. GINA-GOLD. Diagnosis of diseases of chronic airflow limitation: Asthma, COPD and asthma-COPD overlap syndrome (ACOS). 2014. http://www.goldcopd.org/asthmacopd-overlap.html (accessed 24 July 2015).

5. Abdool-Gaffar MS, Ambaram A, Ainslie GM, et al. Guideline for the management of chronic obstructive pulmonary disease - 2011 update. S Afr Med J 2011;101(1):61-73.

6. Koegelenberg CF, Swart F, Irusen EM. Guideline for office 6. Koegelenberg CF, Swart F, Irusen EM. Guideline for office
spirometry in adults, 2012. S Afr Med J 2013;103(1):52-62. [http://dx.doi.org/10.7196/samj.6197]

7. Tashkin DP, Celli B, Decramer M, et al. Bronchodilator 7. Tashkin DP, Celli B, Decramer M, et al. Bronchodilator
responsiveness in patients with COPD. Eur Respir J 2008;31(4):742750. [http://dx.doi.org/10.1183/09031936.00129607]

8. COPD assessment test. http://www.catestonline.org (accessed 24 July 2015).

9. Boggon R, van Staa TP, Timmis A, et al. Clopidogrel discontinuation after acute coronary syndromes: Frequency, predictors and associations with death and myocardial infarction - a hospital registry-primary care linked cohort (MINAP-GPRD). Eur Heart J 2011;32(19):2376-2386.

10. Epworth sleepiness scale. http://yoursleep.aasmnet.org/pdf/ Epworth.pdf (accessed 24 July 2015).

11. Prochaska JJ. Smoking and mental illness - breaking the link. N Engl J Med 2011;365(3):196-198. [http://dx.doi.org/10.1056/

12. Hanania NA, Mullerova H, Locantore NW, et al. Determinants of depression in the ECLIPSE chronic obstructive pulmonary disease cohort. Am J Respir Crit Care Med 2011;183(5):604-611. [http://dx.doi.org/10.1164/rccm.201003-0472OC]

13. Romme EA, Geusens P, Lems WF, et al. Fracture prevention in COPD patients; a clinical 5-step approach. Respir Res 2015;16:32. [http://dx.doi.org/10.1186/s12931015-0192-8] 